\title{
RETÓRICA E OS GÊNEROS ACADÊMICOS: \\ mesclando imagens via analogia para comunicar ciência
}

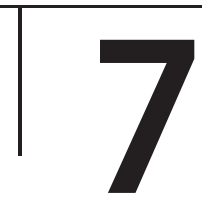

\section{RHETORIC AND THE ACADEMIC GENRE: \\ blending images through analogy to \\ communicate science}

RODRIGUES, Rosana Ferrareto Lourenço

Docente do Instituto Federal de Educação, Ciência e Tecnologia de São Paulo (IFSP) - Câmpus São João da Boa Vista

Doutora em Linguística e Língua Portuguesa pela Unesp - Fclar/Araraquara.

Pós-Doutora pelo Departamento de Ciências Cognitivas da Case Western Reserve University,

Cleveland, Ohio, EUA.

E-mail: rosanaferrareto@ifsp.edu.br

ORCID ID: http://orcid.org/0000-0003-0332-4548

\section{RESUMO:}

Universidades brasileiras têm investido em cursos de redação acadêmica, iniciativa que ajuda graduandos e pesquisadores a desenvolver a escrita para a publicação científica. Entre essas motivações, está a de ensiná-los a argumentar. Das técnicas argumentativas, este trabalho abordará a analogia. Raciocinar por analogia é construir uma estrutura do real que permita encontrar e provar uma verdade graças a uma semelhança de relações (REBOUL, 2004). Resultante da fusão de um elemento do foro com um do tema, é elo no raciocínio indutivo e serve como meio de invenção, ponto de apoio para o pensamento criador (PERELMAN \& OLBRETCHS-TYTECA, 2005). Desencadeia o pensamento, mesclando conceitos aparentemente diversos, para criar o tratável pela mente humana (TURNER, 2014). Na análise de excertos de corpora acadêmicos online, realizadas a partir da ferramenta tecnológica concordanciadora CQPweb, apresentam-se, via analogia, construtos abstratos de diversas áreas, tais como Matemática, Psicologia, Computação e Física. Nos textos analisados, as analogias aparecem através de as if/as though. Apresentar esses marcadores discursivos sob uma abordagem retórica, ao invés de descrever regras gramaticais, promove a criação de imagens de eficaz alcance persuasivo e pode contribuir para o ensino da escrita acadêmica nas universidades. 
Palavras-chave: Retórica. Persuasão. Imagem. Analogia. Gênero Acadêmico.

\section{ABSTRACT:}

Brazilian universities have invested in academic writing courses, an initiative that helps undergraduates and researchers to develop writing for scientific publication. Among these motivations is to teach them to argue. From the argumentative techniques, this work will approach the analogy. To reason by analogy is to construct a structure of the real that allows one to find and prove a truth through a similarity of relationships (REBOUL, 2004). Resulting from the fusion of a forum element and a theme, it is a link in inductive reasoning and serves as a means of invention, a fulcrum for creative thinking (PERELMAN \& OLBRETCHS-TYTECA, 2005). It triggers thinking, merging seemingly diverse concepts, to create the treatable by the human mind (TURNER, 2014). In the analysis of online academic corpora excerpts, conducted through the technological concordance tool CQPweb, we present, by analogy, abstract constructs from various areas, such as Mathematics, Psychology, Computing and Physics. In the parsed texts, analogies emerge in sentences with the transition words as if/as though. Presenting them under a rhetorical approach, rather than describing grammatical rules, promotes the creation of images with effective persuasive reach and can contribute to the teaching of academic writing in universities.

Keywords: Rhetoric. Persuasion. Image. Analogy. Academic Genre.

\section{INTRODUÇÃO}

O número crescente de alunos em cursos de graduação e pósgraduação no Brasil tem feito com que as publicações científicas desses pesquisadores em formação sejam utilizadas como um importante critério de avaliação. Contudo, a escrita acadêmica representa ainda uma grande barreira ao pleno desenvolvimento científico desses pesquisadores. Muitas das razões pelas quais se rejeita um artigo científico para publicação em um periódico de alto impacto estão relacionadas à estrutura argumentativa dos textos. Além disso, indicadores de estudos realizados pelas principais agências de fomento brasileiras revelam que o Brasil está desfavorecido no ranking de publicações (FAPESP, 2011). 
A fim de se conseguir o aceite de um artigo para publicação, é necessário persuadir os pareceristas sobre a relevância da pesquisa e mérito intelectual do trabalho comunicado. Não só a habilidade de promover comunicação clara é importante, mas especialmente a de apresentar a mensagem científica de modo interessante. Nesse sentido, é evidente que a Retórica oferece importantes contribuições para que o pesquisador construa um texto lógico, mas principalmente de alto alcance persuasivo. Entre as estratégias persuasivas de que o pesquisador pode se utilizar está a analogia como recurso de presença. O objetivo deste estudo é verificar, a partir de uma investigação baseada em corpora de textos acadêmicos, como essa técnica argumentativa colabora com a estrutura argumentativa na escrita científica.

\section{PUBLIQUE OU PEREÇA. ESCREVA BEM OU PEREÇA.}

Frente à necessidade de escrever e a importância de publicar, muitos pesquisadores brasileiros têm procurado escritórios especializados de escrita científica ${ }^{1}$. Esse tipo de serviço tem sido oferecido por algumas das principais universidades do Brasil. Marques (2011) afirma que esses cursos e serviços ajudam pesquisadores a redigir um bom trabalho científico - e em inglês, que é a língua da ciência. A ajuda oferecida aos pesquisadores ocorre na forma de workshops promovidos por especialistas, serviços de tradução e revisão e programas de computador capazes de dar forma a artigos científicos. Além disso, muitos especialistas das áreas de exatas e biológicas, tais como Volpato (2013) e Aluísio, Oliveira Júnior e Zucolotto (2014), têm publicado livros que servem como guias de redação a pesquisadores em formação. Essas publicações abordam fundamentos da escrita científica e oferecem modelos linguísticos para a publicação acadêmica. Tanto a proposta dos cursos quanto a dos guias defendem a máxima já consagrada na vida acadêmica, "publique ou pereça".

Contudo, antes de publicar, é preciso escrever bem. "Escreva bem ou pereça" tem sido o novo paradigma para que os pesquisadores consigam a publicação de alto impacto e alcancem, assim, não só o reconhecimento pelo talento, mas a atenção das agências de fomento para suas pesquisas.

1 As propostas de alguns desses escritórios podem ser conhecidas a partir de <http://www.escritacientifica.com/ pt-BR/>; <https://igvec.com> e <http://sbv.ifsp.edu.br/laletec/>. 


\section{ESCREVA BEM OU PEREÇA: CONTRIBUIÇÕES DA NOVA RETÓRICA}

Uma das hipóteses deste trabalho é a de que as figuras retóricas podem contribuir para além da boa estrutura linguística dos textos acadêmicos e do formato esperado para a publicação de alto impacto. Se a publicação de sucesso é, em grande parte, motivada pela boa qualidade dos textos, sua estrutura retórica deve promover o alcance persuasivo da mensagem científica. Nesse sentido, a transmissão de novos conceitos e ideias e de resultados inéditos, que se espera de uma comunicação científica, deve pautar-se no que se entende por presença sob o prisma da Nova Retórica:

Uma das preocupações do orador será tornar presente, apenas pela magia de seu verbo, o que está efetivamente ausente e que ele considera importante para a sua argumentação, ou valorizar, tornando mais presentes, certos elementos efetivamente oferecidos à consciência. [...] Aplicar a razão à imaginação para melhor mover a vontade é essencialmente vinculado aos efeitos de presença" (PERELMAN \& OLBRECHTS-TYTECA, 2005, p. 133).

Valorizar, tornando mais presentes, as importantes contribuições de uma investigação científica inédita e relevante é uma atividade que pode ser entendida como fazer persuasivo. Persuadir o auditório do valor de verdade atribuído a um novo saber, transmitido pelo escritor-cientista, deve emergir da organização textual de um esquema argumentativo que vai além das categorias canônicas da argumentação (premissa-hipótese, justificativa, materiais e métodos, resultados obtidos e conclusão) na dimensão comunicativa. É necessário ir além do convencimento porque, "por não ser verdade, o saber construído pelo cientista nunca é definitivo e sim, provisório, embora, de forma dominante, o argumentativo científico afirma a veracidade do saber comunicado" (SILVEIRA, 2012, p. 48).

A contribuição da Nova Retórica para a comunicação do verossímil pode ser encontrada no uso da analogia. Raciocinar por analogia é construir uma estrutura do real que permita encontrar e provar uma verdade graças a uma semelhança de relações (REBOUL, 2004). Resultante da fusão de um elemento do foro com um do tema, a analogia é elo no raciocínio indutivo, serve como meio de 
invenção, ponto de apoio para o pensamento criador (PERELMAN \& OLBRETCHS-TYTECA, 2005). Por exemplo, se digo que a publicação científica de alto impacto constante está para a vida como a ausência de publicação está para a morte, tenho uma analogia proporcional aristotélica que comunica a ideia de que é preciso publicar para obter sucesso e manter-se ativo na carreira acadêmica.

Um outro exemplo do alcance persuasivo da analogia foi empregado em:

Escrever requer uma organização que permita ao leitor acompanhar as ideias do texto, do início ao fim. Uma receita de bolo, por exemplo, permite ao leitor seguir um processo em dada ordem. Primeiro, instrui a preparar a lista de ingredientes e suas medidas. Depois, especifica o procedimento para mexer os ingredientes até os passos finais, depois dos quais o bolo estará pronto para ser consumido. Os documentos escritos devem alcançar o mesmo contínuo que a receita para atrair a atenção do leitor. [...] Uma publicação de sucesso consiste em mostrar ao leitor como as ideias surgiram, como numa receita de bolo, em que se listam os ingredientes e que termina como um bolo quente saindo do forno (OLIVEIRA JÚNIOR et al, 2014, p. 18-19).

Essa analogia, motivada pelas metáforas bolo: texto; ingredientes e suas medidas: materiais e métodos; receita de bolo: documento escrito, apresenta a publicação de sucesso como um bolo quente saindo do forno. Alcançar o mesmo contínuo que a "receita" para atrair a atenção do leitor requer organização escrita.

\section{MESCLANDO IMAGENS VIA ANALOGIA PARA COMUNICAR CIÊNCIA}

A imagem do bolo quente saindo do forno remete, na analogia construída por Oliveira Júnior et al (2014), à imagem de um artigo publicado em periódico de alto impacto. Por ser uma técnica argumentativa fundada na estrutura do real, a analogia tem efeito persuasivo na interação entre tema e foro, acentuando a ação do tema - a publicação (valor intangível) - e transferindo o valor do foro - o bolo (valor tangível) $\neg-$ para o tema. Nesse exemplo, "o foro é tomado de empréstimo ao domínio sensível e o tema ao domínio espiritual" (PERELMAN \& OLBRETCHS-TYTECA, 2005, p. 434). Isso confere à 
analogia um status de técnica discursiva que permitem provocar ou aumentar a adesão dos espíritos às teses que se apresentam ao seu assentimento (PERELMAN \& OLBRETCHS-TYTECA, 2005), o que atesta seu caráter persuasivo.

Além disso, o caráter didático e ilustrativo da analogia se deve ao fato de que, segundo Bergen (2012), talvez entendamos a língua por meio de simulações mentais, o que seria como experimentar as coisas que a língua descreve. Diferentes construções argumentativas realmente parecem contribuir para o significado a ser simulado mentalmente. É evidente que a analogia é ainda mais apta a fornecer simulações do que se pensava (BERGEN, 2012). Isso porque, de acordo com Turner (2014), a analogia desencadeia o pensamento, mesclando conceitos aparentemente diversos (pesquisar, escrever, publicar), para criar o tratável pela mente humana (a imagem do bolo). Conceber um texto publicado como um bolo pronto é possível porque a analogia é entendida como uma forma natural do pensamento humano.

Sob o prisma da Linguística Cognitiva, graças à Teoria da Integração Conceptual ${ }^{2}$, também conhecida como mesclagem ou blend, via analogia, entende-se construtos abstratos apresentando-os a partir de elementos concretos. Essa operação cognitiva, humana por excelência, é que dá origem às ideias, graças à criatividade humana. A criatividade consiste em fazer a união entre dois inputs, gerando um espaço blend, e dando origem às novas ideias (TURNER, 2014).

$\mathrm{O}$ bolo quente saindo do forno simulando o texto acabando de ser publicado em um periódico de alto impacto é possível graças ao estatuto da analogia - não aquela analogia aristotélica matemática, em que a proporcionalidade entre os elementos associados devia ser simétrica. Segundo Hofstadter \& Sander (2013), é na busca por analogias fortes e provocadoras em nossa memória que tentamos extrair a essência de situações não familiares que enfrentamos o tempo todo. A busca por analogias apropriadas é um tipo de arte que merece o rótulo de 'vital'. No esquema do blending criativo, teríamos a representação dessa mesclagem conforme apresenta a Figura 1.

2 Essa teoria foi apresentada pela primeira vez em $\neg$ Fauconnier, G. \& Turner, M. The way we think: conceptual blending and the mind's hidden complexities. New York: Basic Books, 2002. 
Figura 1 - Mesclagem de texto científico como uma receita de bolo

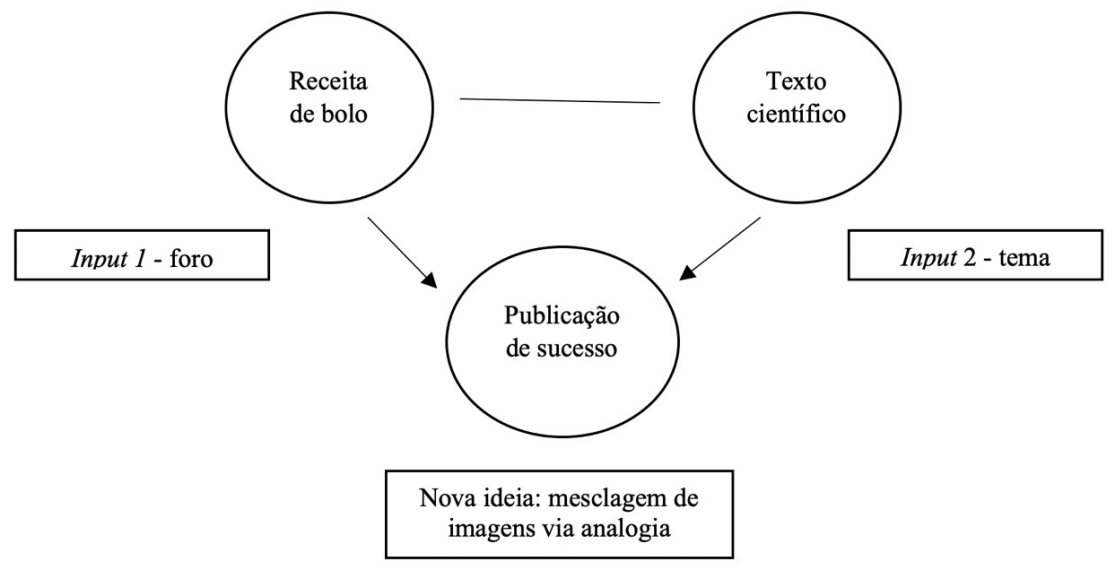

Fonte: Elaboração própria baseada em Oliveira Júnior (2014)

A mesclagem de imagens via analogia nos gêneros acadêmicos foi investigada, para este trabalho, em corpora de artigos científicos de várias áreas do conhecimento e a análise revelou que, graças à analogia, apresentam-se construtos teóricos abstratos a partir de simulações e metáforas que facilitam seu entendimento.

OCORRÊNCIA DE ANALOGIA NA ESCRITA ACADÊMICA: UMA PROPOSTA DE DESCRIÇÃO DE DADOS EM ARTIGOS CIENTÍFICOS

A Linguística de Corpus (LC) foi o expediente metodológico empregado para a investigação sobre o uso de analogia nos artigos científicos. A LC é uma área de pesquisa inserida na esfera da Linguística Aplicada que se ocupa da coleta e exploração de corpora, ou conjuntos de dados linguísticos textuais que foram coletados criteriosamente com o propósito de servirem para a pesquisa de uma língua ou variedade linguística. Como tal, dedica-se à exploração da linguagem através de evidências empíricas, extraídas por meio de computador (BERBER SARDINHA, 2004).

A respeito da eficácia de se utilizar a LC em pesquisas sobre a escrita científica, Tagnin (2014) defende que um corpus customizado em dado domínio do conhecimento, se propriamente compilado, deve apresentar um número significativo de itens convencionais que o caracterizam. Para este estudo, nosso intuito é verificar se a analogia é característica de textos científicos, tarefa de demanda geral para a qual não foi necessário compilar um corpus especializado. 
Os textos foram coletados de uma base de dados online, o CQPWeb, disponível gratuitamente em <cqpweb.lancs.ac.uk/>33, criado e gerenciado pela Universidade de Lancaster, no Reino Unido. O CQPWeb é um software concordanciador ${ }^{4}$ online com um corpus de referência ${ }^{5}$ embutido. A interface desse software pode ser visualizada na Figura 2.

Figura 2 - Interface da CQPWeb

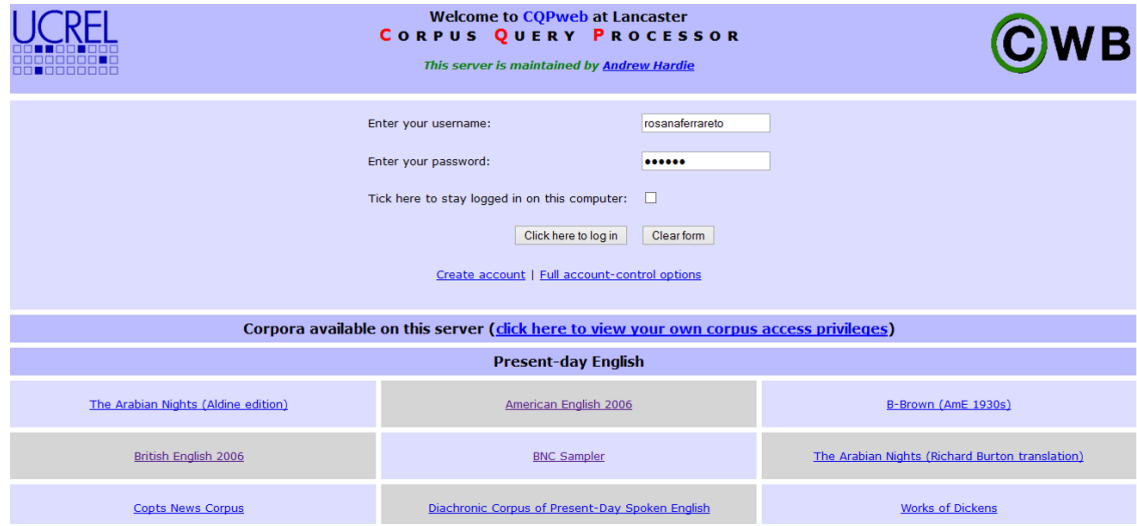

Fonte: <cqpweb.lancs.ac.uk>

Para o levantamento de dados, o gênero selecionado foi o learned, que corresponde ao acadêmico, em dois corpora: um de inglês americano (American English 2006) e um de inglês britânico (British English 2006). Para verificar as ocorrências de analogias nos artigos científicos foram usadas como palavras de busca "as if" e "as though" ("como se"), que são marcadores discursivos que introduzem comparações/analogias.

O resultado das buscas no corpus de inglês americano para as if foi de sete ocorrências em seis textos diferentes, com uma frequência de 37.73 instâncias por milhão de palavras, enquanto que para as though, o corpus retornou três ocorrências para três textos diferentes com uma frequência de 16.17 instâncias por milhão de palavras. Esses resultados podem ser visualizados na Figura 3.

3 Tutoriais sobre o uso do CQPWeb podem ser obtidos a partir de $<$ www.youtube.com/watch?v=Yf1 KxLOI8z8 $>$. 4 Concordanciador: programa que extrai todas as ocorrências de uma palavra de busca num corpus juntamente com seu cotexto, apresentando-as na forma de uma concordância (TAGNIN, 2010).

5 Corpus de referência: corpus que serve de termo de comparação para o corpus de estudo. Em geral, deve ter três a cinco vezes o tamanho do corpus de estudo (TAGNIN, 2010). Neste trabalho, estamos utilizando o corpus de referência como corpus de estudo, uma vez que nosso intuito não é uma análise comparativa, por exemplo, entre artigos científicos de duas línguas. 
Figura 3 - Ocorrências de analogias em corpus acadêmico de inglês americano

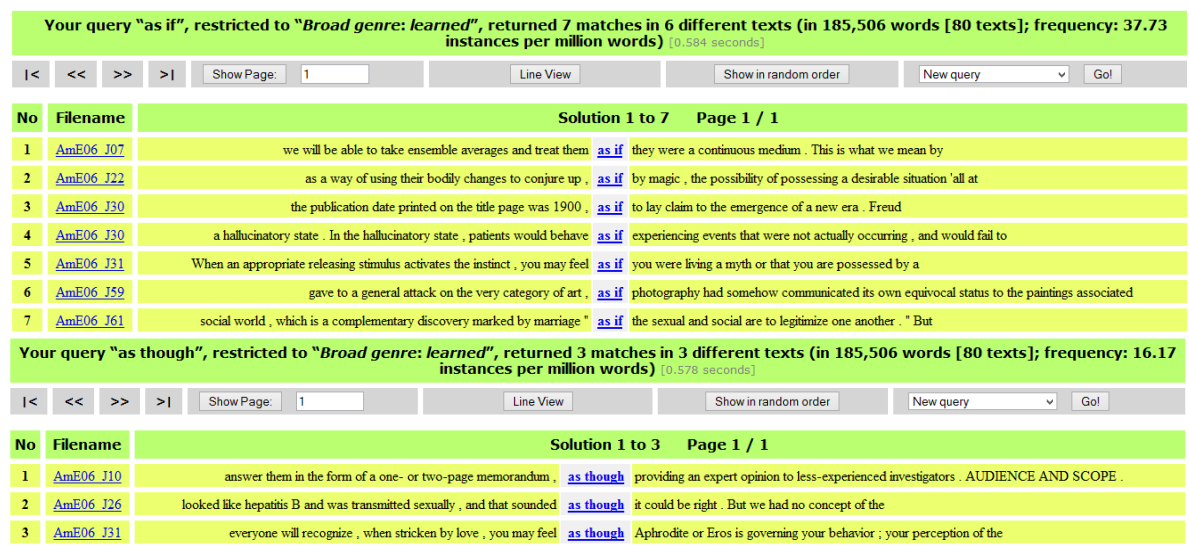

Fonte: <cqpweb.lancs.ac.uk>

O resultado das buscas no corpus de inglês britânico para as if foi de duas ocorrências em dois textos diferentes, com uma frequência de 10.99 instâncias por milhão de palavras, enquanto que para as though, o corpus retornou três ocorrências para dois textos diferentes com uma frequência de 16.48 instâncias por milhão de palavras. Esses resultados podem ser visualizados na Figura 4.

Figura 4 - Ocorrências de analogias em corpus acadêmico de inglês britânico

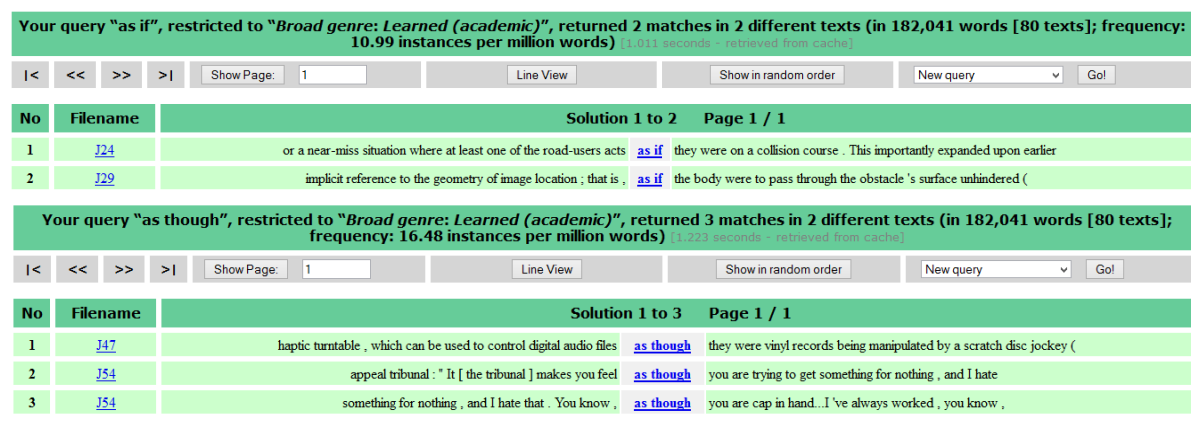

Fonte: <cqpweb.lancs.ac.uk>

Nosso intuito não é delinear quantitativamente a presença de analogias no discurso acadêmico, mais especificamente, nos artigos científicos, mas os dados atestam a presença dessa técnica argumentativa nesses tipos de textos.

O que nos interessa nesta investigação é verificar como a analogia é empregada para nos textos científicos em diversas áreas 
do conhecimento e compreender como esse emprego contribui para o alcance argumentativo desses textos. Por isso, apresentamos alguns desses dados a seguir, no Quadro 1.

Quadro 1 - Dados do corpus com o emprego da analogia

\begin{tabular}{|c|c|c|c|}
\hline $\begin{array}{c}\text { ÁREA DO } \\
\text { CONHECIMENTO }\end{array}$ & $\begin{array}{c}\text { ENUNCIADO DO } \\
C O R P U S\end{array}$ & $\begin{array}{l}\text { TRADUÇÃO } \\
\text { PARA O } \\
\text { PORTUGUÊS }\end{array}$ & $\begin{array}{l}\text { EFEITO DA } \\
\text { ANALOGIA }\end{array}$ \\
\hline Matemática & $\begin{array}{l}\text { The geometry of } \\
\text { image location } \\
\text { works as if the } \\
\text { body were to } \\
\text { pass through an } \\
\text { obstacle. }\end{array}$ & $\begin{array}{l}\text { A geometria de } \\
\text { localização de } \\
\text { imagem funciona } \\
\text { como se o corpo } \\
\text { fosse para passar } \\
\text { através de um } \\
\text { obstáculo. }\end{array}$ & $\begin{array}{l}\text { Explica-se } \\
\text { geometria pelo } \\
\text { movimento do } \\
\text { corpo passando por } \\
\text { um obstáculo. }\end{array}$ \\
\hline Psicologia & $\begin{array}{l}\text { A conflict is an } \\
\text { event that would } \\
\text { have led to a } \\
\text { collision if both } \\
\text { road-users had } \\
\text { continued as if } \\
\text { they were on a } \\
\text { collision course. }\end{array}$ & $\begin{array}{l}\text { Um conflito é um } \\
\text { evento que teria } \\
\text { levado a uma } \\
\text { colisão se ambos } \\
\text { os usuários da } \\
\text { estrada tivessem } \\
\text { seguido como } \\
\text { se estivessem } \\
\text { em uma rota de } \\
\text { colisão. }\end{array}$ & $\begin{array}{l}\text { Conflitos sociais } \\
\text { são como a colisão } \\
\text { de um carro. }\end{array}$ \\
\hline Computação & $\begin{array}{l}\text { Programming- } \\
\text { like interaction } \\
\text { techniques control } \\
\text { digital audio files } \\
\text { as though they } \\
\text { were vinyl records } \\
\text { manipulated by a } \\
\text { scratch disc jockey. }\end{array}$ & $\begin{array}{l}\text { Técnicas de } \\
\text { interação como } \\
\text { programação } \\
\text { controlam } \\
\text { arquivos de áudio } \\
\text { digital como se } \\
\text { fossem discos de } \\
\text { vinil manipulados } \\
\text { por um disc } \\
\text { jockey. }\end{array}$ & $\begin{array}{l}\text { Técnicas de } \\
\text { programação } \\
\text { funcionam como o } \\
\text { trabalho de um DJ. }\end{array}$ \\
\hline Física & $\begin{array}{l}\text { Gas consists of } \\
\text { a collection of } \\
\text { myriads of particles } \\
\text { that execute } \\
\text { collective motions } \\
\text { treated as if they } \\
\text { were a continuous } \\
\text { medium. }\end{array}$ & $\begin{array}{l}\text { O gás é composto } \\
\text { por uma coleção } \\
\text { de miríades } \\
\text { de partículas } \\
\text { que executam } \\
\text { movimentos } \\
\text { coletivos tratados } \\
\text { como se fossem } \\
\text { um contínuo. }\end{array}$ & $\begin{array}{l}\text { Partículas } \\
\text { astrofísicas } \\
\text { são vistas num } \\
\text { contínuo. }\end{array}$ \\
\hline
\end{tabular}

Fonte: Elaboração própria 
Os efeitos das analogias empregadas para apresentar construtos teóricos a partir de temas especializados, à primeira vista, ininteligíveis para o público leitor dos artigos científicos, constitui-se como técnica argumentativa com potencial alcance persuasivo, uma vez que aproxima leitor e escritor-cientista exatamente por romper o distanciamento ocasionado pela incompreensão do texto. Entre outras estratégias retóricas, o uso da analogia está à mercê da argumentação e trata-se de uma escolha do pesquisador entre ser louvado e aplaudido pelos pares da Academia, produzindo textos barrocos e intimidativos, ou escolher ter mais leitores e disponibilizar a eles um alimento intelectual mais nutritivo e saboroso, como defende Abreu (2015).

\section{CONSIDERAÇÕES FINAIS}

Além dos resultados apresentados na investigação proposta ocorrência de analogias na escrita científica - a principal contribuição deste estudo é a reflexão de que apresentar os marcadores discursivos as if/as though sob uma abordagem retórica, ao invés de meramente descrevê-los como regras gramaticais, promove a criação de imagens, via analogia, de eficaz alcance persuasivo. Essa abordagem pode contribuir para o ensino de redação científica nas universidades.

Outra contribuição é a de integrar aos estudos retóricos os arcabouços teóricos da Linguística Cognitiva para pensar não só sobre os efeitos da analogia no discurso persuasivo, mas também para compreender como a analogia emerge do pensamento humano e se materializa na língua.

É também relevante considerar o emprego da Linguística de Corpus como expediente metodológico em estudos sobre o letramento acadêmico de pesquisadores em formação e sua produção escrita. E é então que se abrem novas perspectivas de investigação da escrita científica: verificar se a analogia é também uma técnica argumentativa adotada nos textos científicos publicados em língua portuguesa; que áreas do conhecimento a empregam com mais frequência, como e por que; e qual a disposição da analogia na composição do discurso retórico presente nos textos acadêmicos. 


\section{REFERÊNCIAS}

ABREU, A. S. A linguagem do texto científico: algumas críticas e sugestões. In: LUCAS, O. P.; RODRIGUES, R. F. L. (orgs.) Temas e Rumos nas Pesquisas em Linguística (Aplicada): Questões empíricas, éticas e práticas. Vol. 1. Campinas: Editora Pontes, 2015. (no prelo) ALUÍSIO, S. M.; OLIVEIRA JÚNIOR., O. N.; ZUCOLOTTO, V. Models for scientific writing. In: SCHUSTER, Ethel; LEVKOWITZ, Haim; OLIVEIRA JR, Osvaldo N. (Orgs.). Writing scientific papers in English successfully: your complete roadmap. Hyprtek: Andover and São Carlos, 2014. p. 30-58.

BERBER SARDINHA, T. Linguística de Corpus. Barueri/SP: Manole, 2004.

BERGEN, B. K. Louder than words: the new science of how the mind makes meaning. New York: Basic Books, 2012.

FAPESP. Indicadores de Ciência, Tecnologia e Inovação em São Paulo. 2010. Coordenação geral: Ricardo Renzo Brentani; Carlos Henrique de Brito Cruz. Coordenação executiva: Wilson Suzigan, João Eduardo de Morais Pinto Furtado, Renato de Castro Garcia. São Paulo: FAPESP, 2011.

HOFSTADTER, D.; SANDER, E. Surfaces and essences: analogy as the fuel and fire of thinking. New York: Basic Books, 2013.

LUCAS, O. P.; RODRIGUES, R. F. L. (orgs.) Temas e Rumos nas Pesquisas em Linguística (Aplicada): Questões empíricas, éticas e práticas. Vol. 1. Campinas: Editora Pontes, 2015. (no prelo)

MARQUES, F. Escreva bem ou pereça: Cursos e serviços ajudam pesquisadores a redigir um bom trabalho científico. Pesquisa Fapesp. ed. 182. abr. p. 34-39. 2011. Disponível em: <http://revistapesquisa.fapesp.br/wp-content/uploads/2011/04/034-039-182.pdf>. Acesso em: 05 Jun. 2015.

OLIVEIRA JÚNIOR, O. N.; SCHUSTER, E.; LEVKOWITZ, H.; ZUCOLOTTO, V. The fundamentals of scientific writing. In: SCHUSTER, E.; LEVKOWITZ, H.; OLIVEIRA JÚNIOR, O. N. (Orgs.). Writing scientific papers in English successfully: your complete roadmap. Hyprtek: Andover and São Carlos, 2014. p. 18-28.

PERELMAN, C.; OLBRETCHS-TYTECA, L. Trad. Maria Ermantina de Almeida Prado Galvão. Tratado da argumentação: a nova retórica. 2. ed. São Paulo: Martins Fontes, 2005.

REBOUL, O. Trad. Ivone Castilho Benedetti. Introdução à retórica. 2. ed. São Paulo: Martins Fontes, 2004.

SCHUSTER, E.; LEVKOWITZ , H.; OLIVEIRA JR, O. N. (Orgs.). Writing scientific papers in English successfully: your complete roadmap. Hyprtek: Andover and São Carlos, 2014.

SILVEIRA, R. C. P. Textos do discurso científico: pesquisa, revisão e ensaio. São Paulo: Terracota, 2012.

TAGNIN, S. E. O. Using Corpus Linguistics to overcome the language barrier. In: SCHUSTER, E.; LEVKOWITZ, H.; OLIVEIRA JR, O. N. (Orgs.). Writing scientific papers in English successfully: your complete roadmap. Hyprtek: Andover and São Carlos, 2014. p. 84-114. 
TAGNIN, S. E. O. e VIANA, V. (orgs.). Corpora no Ensino de Línguas Estrangeiras. São Paulo: Hub Editorial, 2010.

TAGNIN, S. E. O. Glossário de linguística de corpus. In: TAGNIN, S. E. O. e VIANA, V. (orgs.). Corpora no Ensino de Línguas Estrangeiras. São Paulo: Hub Editorial, 2010. Disponível em < http://www.hubeditorial.com.br/site/recursos/5_glossario/glossario_423. pdf $>$. Acesso em: 17 jul. 15.

TURNER, M. The origin of ideas: blending, creativity and the human spark. Oxford: Oxford University Press, 2014.

VOLPATO, G. Ciência: da filosofia à publicação. 6 ed. Edição Cultura Acadêmica, 2013.

Enviado em: $\quad$ 05/12/2019

Aceite em: $\quad$ 18/12/2019 